\title{
Differential association of frailty with cognitive decline and sarcopenia in community-dwelling older adults.
}

\section{AUTHOR(S):}

Nishiguchi, Shu; Yamada, Minoru; Fukutani, Naoto; Adachi, Daiki; Tashiro, Yuto; Hotta, Takayuki; Morino, Saori; ... Arai, Hidenori; Tsuboyama, Tadao; Aoyama, Tomoki

\section{CITATION:}

Nishiguchi, Shu ...[et al]. Differential association of frailty with cognitive decline and sarcopenia in community-dwelling older adults.. Journal of the American Medical Directors Association 2015, 16(2): 120-124

\section{ISSUE DATE:}

2015-02-01

URL:

http://hdl.handle.net/2433/196037

\section{RIGHT:}

(c) 2015 AMDA - The Society for Post-Acute and Long-Term Care Medicine. NOTICE: this is the author's version of a work that was accepted for publication in Journal of the American Medical Directors Association. Changes resulting from the publishing process, such as peer review, editing, corrections, structural formatting, and other quality control mechanisms may not be reflected in this document. Changes may have been made to this work since it was submitted for publication. A definitive version was subsequently published in Journal of the American Medical Directors

Association, 16(2), 2015, doi:10.1016/j.jamda.2014.07.010; この論文は出版社版でありません。引用の際には出版社版を ご確認ご利用ください。; This is not the published version. Please cite only the published version. 
1 Differential association of frailty with cognitive decline and sarcopenia in

2 community-dwelling older adults

3

$4 \quad$ Running Title: Frailty with cognitive decline and sarcopenia

6 Authors

7 Shu Nishiguchi, ${ }^{\mathrm{a}, \mathrm{b}}$ Minoru Yamada, ${ }^{\mathrm{c}}$ Naoto Fukutani, ${ }^{a}$ Daiki Adachi, ${ }^{\mathrm{a}}$ Yuto Tashiro, ${ }^{\text {a }}$ Takayuki Hotta, ${ }^{\mathrm{a}}$

8 Saori Morino, ${ }^{\text {a }}$ Hidehiko Shirooka, ${ }^{\text {a }}$ Yuma Nozaki, ${ }^{a}$ Hinako Hirata, ${ }^{\text {a }}$ Moe Yamaguchi, ${ }^{\text {a }}$ Hidenori Arai, ${ }^{a}$

$9 \quad$ Tadao Tsuboyama, $^{\text {a }}$ and Tomoki Aoyama ${ }^{\mathrm{a}}$

\section{Affiliations}

12 a. Department of Human Health Sciences, Graduate School of Medicine, Kyoto University, Kyoto, 13 Japan

14 b. Japan Society for the Promotion of Science, Tokyo, Japan

15 c. Graduate School of Comprehensive Human Sciences, University of Tsukuba, Tokyo, Japan

17 Corresponding author:

18 Shu Nishiguchi, R.P.T., M.Sc.

19 Department of Physical Therapy, Human Health Sciences, Graduate School of Medicine, Kyoto 20 University, Kyoto, Japan

2153 Kawahara-cho, Shogoin, Sakyo-ku, Kyoto 606-8507, Japan

22 E-mail: nishiguchi.shu.82s@st.kyoto-u.ac.jp 
$1 \quad$ Phone: +81-75-751-3935

$2 \quad$ Fax: $+81-75-751-3909$

3

4 Keywords: Frailty, cognitive decline, sarcopenia, community-dwelling older adults 
1 Abstract:

2 Objectives: Frailty in older adults is a serious problem due to various adverse health outcomes in

3 many countries with aging populations, such as Japan. The purpose of this study was to determine

4 whether frailty and pre-frailty are associated with cognitive decline and sarcopenia in

5 community-dwelling older adults.

6 Design: This is a cross-sectional study.

7 Setting: Japan.

$8 \quad$ Participants: The participants were 273 Japanese community-dwelling older women aged 65

9 years and older.

10 Measurements: We used the frailty criteria developed by the Cardiovascular Health Study to

11 define physical frailty. We divided the cohort into non-frail, pre-frail, and frail according to frailty

12 scores. Cognitive decline and memory decline were defined by using the Mini-Mental State

13 Examination and Scenery Picture Memory Test, respectively. Sarcopenia was defined according to

14 the diagnostic algorithm recommended by the Asian Working Group for Sarcopenia.

15 Results: In the multivariate logistic regression analysis by using non-frail participants as the 16 reference, pre-frail elderly individuals were significantly more likely to have sarcopenia than

17 non-frail elderly individuals (odds ratio [OR]: 2.77, 95\% confidence interval [Cl]: 1.05-9.26), but not 18 cognitive decline or memory decline. Frail elderly individuals were significantly more likely to have 19 cognitive decline (OR: 5.76, 95\% Cl: 1.20-27.6), memory decline (OR: 5.53, 95\% Cl: 1.64-18.7) 20 and sarcopenia (OR: 19.1, 95\% Cl: 3.73-98.0) than non-frail elderly individuals.

21 Conclusions: Sarcopenia was associated with pre-frailty and frailty, while cognitive decline was 
1 associated only with frailty. 


\section{Highlights}

2 - We studied the association of physical frailty with cognitive decline and sarcopenia.

3 - We focused on the level of frailty in community-dwelling older adults.

4 - Frailty, but not pre-frailty, was associated with cognitive and memory decline.

$5 \quad \cdot$ Both frailty and pre-frailty were associated with sarcopenia. 


\section{Introduction}

2 Frailty in older adults is a serious concern in countries with aging populations, such as Japan. In

3 general, frailty is defined as a vulnerable state that places older adults at high risk for adverse

4 health outcomes, such as falls, hospitalization, and mortality ${ }^{1,2}$. Using the frailty criteria developed

5 by the Cardiovascular Health Study (CHS), the overall prevalence of frailty in community-dwelling

6 adults aged 65 or older in the United States has been found to range from $7 \%$ to $12 \%$, and was

7 greater in women than in men ${ }^{1}$. In Japanese, the prevalence of frailty in community-dwelling adults

8 aged 65 or older was $11.3 \%$, and it increased with aging ${ }^{3}$. Frail older adults are considered to have

9 a substantially increased risk of disability, dependency, and need for long-term care insurance.

10 Therefore, prevention and early detection of frailty is important for addressing age-related health

11 care issues.

The causes of frailty are not clearly defined, but it has been suggested that age-related physical changes are the main causes of frailty ${ }^{4}$. Sarcopenia, defined as progressive loss of skeletal muscle mass, strength, and physical function, is regarded as a key component of physical frailty ${ }^{5,6}$. The Interventions on Frailty Working Group assessed various methods for screening, recruiting, evaluating, and retaining frail elderly individuals in clinical trials ${ }^{7}$. They reported that most

researchers focused on the following domains when identifying physical frailty: mobility, such as

lower-extremity performance and gait abnormalities; muscle weakness; poor exercise tolerance;

unstable balance; and factors related to body composition, such as weight loss, malnutrition, and 
1 increases in inflammatory cytokines. ${ }^{5}$ Thus, the domains of frailty overlap with the factors related to

2 sarcopenia, and both frailty and sarcopenia mutually result in adverse health outcomes 5,6 .

3 Of note, some definitions of frailty include cognitive function and dementia ${ }^{4,8}$. Several

4 cross-sectional studies have reported an association between physical frailty and cognitive function

$5 \quad 1,7,9,10$. In addition, longitudinal studies have revealed that a higher level of physical frailty is

6 associated with increased risk of incident Alzheimer's disease (AD) ${ }^{11}$ and mild cognitive impairment

$7 \quad(\mathrm{MCl})^{12}$. It has been indicated that frailty is associated with AD pathology ${ }^{13}$ and its biological

8 mechanisms ${ }^{14}$. However, not all dementia patients become frail; therefore, the association

9 between frailty and cognitive impairment warrants further study.

10 Frailty is associated with sarcopenia and cognitive decline. Furthermore, frailty has been

11 considered to include other aspects, such as psychosocial issues and comorbidities ${ }^{15}$. However, it 12 is unclear whether the associations between frailty and cognitive decline as well as between frailty

13 and sarcopenia are different according to the level of frailty. Therefore, the purpose of this study

14 was to determine whether frailty and pre-frailty are associated with cognitive decline and

15 sarcopenia in community-dwelling older adults. 


\section{Methods}

\section{Participants}

$3 \quad$ Participants for this study were recruited through the local press; 273 Japanese women aged 65

4 years and older (mean age $73.0 \pm 5.4$ years) responded. We included community-dwelling older

5 adults who were independent in activities of daily living (ADLs). Participants were interviewed and

6 excluded if they met any of the following criteria: severe cardiac, pulmonary, or musculoskeletal

7 disorders; severe neurological disorders, such as Parkinson disease and stroke; and participation

8 in Japan's long-term care service. The following data were collected from each participant: age,

9 height, weight, and number of medications being consumed.

10 Written informed consent was obtained from each participant in accordance with the guidelines

11 approved by the Kyoto University Graduate School of Medicine and the Declaration of Human 12 Rights, Helsinki, 1975. The study protocol was approved by the ethical committee of the Kyoto

13 University Graduate School of Medicine.

\section{Assessment of frailty}

16 We measured physical frailty domains determined in a previous study ${ }^{3}$. As in that study, we 17 considered the frailty phenotype to be characterized by limitations in the following five domains by 18 using frailty criteria developed by the CHS ${ }^{1}$ : slowness, weakness, exhaustion, low activity, and 19 shrinking. To measure slowness, each participant's 10-m normal walking speed (m/s) was 20 calculated, and a slow walk was defined as $<1.0 \mathrm{~m} / \mathrm{s}$. To measure weakness, low grip strength was 21 established according to a sex-specific cutoff of the average grip strength in each arm (women: 
$1<17 \mathrm{~kg}$ ). Exhaustion was assessed via self-report by using the Geriatric Depression Scale ${ }^{16}$-i.e.,

2 exhaustion was defined as a negative ("no") answer to the question "do you feel full of energy?" We

3 evaluated the role of physical activity by asking the following questions about time spent engaged

4 in sports and exercise: (1) "Do you engage in moderate levels of physical exercise or sports aimed

$5 \quad$ at health?" and (2) "Do you engage in low levels of physical exercise aimed at health?" If a

6 participant answered "no" to both of these questions, then we considered their physical activity to

7 be low. Shrinking was established according to self-reports of weight loss in response to the

8 following question: "In the past 2 years, have you lost more than $5 \%$ of your body weight

9 irrespective of intent to lose weight?" If a participant answered "yes" to this question, then we 10 considered them to have shrunk. We calculated the number of affected domains and classified

11 participants as follows: pre-frailty $=1$ or 2 , frailty $\geq 3^{1}$.

\section{Measurement of cognitive function}

Mini-Mental State Examination (MMSE) ${ }^{17}$ and the Scenery Picture Memory Test (SPMT) ${ }^{18}$.

16 Global cognitive function was assessed by using the MMSE, a standard test in cognitive aging

17 research to assess mental status. The MMSE tests five areas of cognitive function: orientation,

18 registration, attention and calculation, recall, and language. It has 11 questions and a possible

19 maximum score of 30 . We divided the participants into a normal or a cognitive decline group based

on a cut-off of $23 / 24$ as the MMSE score ${ }^{19}$.

The SPMT is a simple memory test that assesses visual memory combined with verbal 
1 responses. This test uses a line drawing of a living room in a house with 23 objects commonly

2 observed in daily life on an A4 piece of paper. The examinee is instructed to look at the picture for 1

3 minute and remember the items. After this encoding period, participants are distracted by

4 completing a brief digits forward test. Participants are then asked to recall the objects in the picture

$5 \quad$ without a time limitation. The recall usually takes approximately 2 minutes. The number of items

6 recalled is the score for the SPMT. We divided the participants into a normal or memory decline

7 group based on a cut-off of 9/10 as the SPMT score ${ }^{18}$.

\section{$9 \quad$ Definition of sarcopenia}

We defined sarcopenia by using the diagnostic algorithm recommended by the Asian Working

11 Group for Sarcopenia (AWGS), which assesses the presence of both low muscle function (low physical performance or low muscle strength) and low muscle mass ${ }^{20}$. A bioelectrical impedance data acquisition system (Inbody 430; Biospace Co, Ltd, Seoul, Korea) was used to perform bioelectrical impedance analysis ${ }^{21}$. This system uses electrical current at multiple frequencies (5, $50,250,500$, and $1000 \mathrm{kHz}$ ) to directly measure the amount of extracellular and intracellular water. Participants stood on two metallic electrodes and held metallic grip electrodes. Using segmental body composition, appendicular skeletal muscle mass was determined and used for further analysis. Skeletal muscle mass index (SMI) was calculated by dividing muscle mass by height 19 squared in meters $\left(\mathrm{kg} / \mathrm{m}^{2}\right)$. This index has been used in several epidemiological studies ${ }^{22,23}$. If a 20 participant had both low muscle function (slow walking speed, $\leq 0.8 \mathrm{~m} / \mathrm{s}$; low grip strength for 21 women, $\leq 18 \mathrm{~kg}$ ) and low SMI (low muscle mass for women, $\leq 5.7 \mathrm{~kg} / \mathrm{m}^{2}$ ), then they were defined as 
1 having sarcopenia 20 .

$3 \quad$ Statistical analysis

4 Prior to the analysis, we classified participants into the following three groups according to their

5 frailty score: non-frailty, pre-frailty, and frailty. Differences in the demographic variables, MMSE,

6 SPMT, and SMI among the three groups were examined by using the analysis of variance (ANOVA).

$7 \quad$ When a significant effect was found, differences were determined with the Tukey-Kramer post-hoc

8 test. Differences in the prevalence of cognitive decline, memory decline, and sarcopenia among the

9 three groups were evaluated by using the chi-square test. In addition, multivariate logistic

10 regression analyses, adjusted for age, BMI, and medications, were performed to determine

11 whether physical frailty was associated with cognitive decline, memory decline, or sarcopenia. For

12 this analysis, cognitive decline, memory decline, and sarcopenia were dependent variables, while

13 the three frailty groups (dummy coded with non-frailty group as the reference group) were

14 independent variable. Subsequent multivariate logistic regression analyses were performed to

15 determine the independent association between each level of frailty and the risk of cognitive

16 decline or sarcopenia. In these subsequent analyses (adjusted for age and medications), the frailty

17 groups were the dependent variables, and cognitive decline and sarcopenia were independent

18 variables. Odds ratios (ORs) with 95\% confidence intervals $(\mathrm{Cl})$ were presented. Statistical

19 analyses were carried out by using SPSS Statistics for Windows, version 20.0 (SPSS Inc. Chicago,

IL, USA), with a significance threshold of 0.05 . 
$1 \quad$ Results

2 Demographic data for participants stratified by frailty group are shown in Table 1 . There were 89

3 participants (32.6\%) in the non-frailty group, 155 participants (56.8\%) in the pre-frailty group, and

429 participants $(10.6 \%)$ in the frailty group. ANOVA showed that there were significant differences in

5 age, walking speed, and grip strength among the three groups (Table 1). In the chi-square test,

6 there were significant differences in the prevalence of cognitive decline, memory decline, and

7 sarcopenia (Table 1$)$. In addition, the frailty group had significantly lower MMSE $(F=6.78, p=$

$8 \quad 0.001$, Figure $1 \mathrm{a})$ and SPMT $(F=18.5, p<0.001$, Figure $1 \mathrm{~b})$ than the non-frailty and pre-frailty

9 groups, and lower SMI ( $F=5.17, p=0.006$, Figure $1 c)$ than the non-frailty group.

Eighteen participants (6.6\%) had cognitive decline, 20 participants (7.3\%) had memory decline,

and 23 participants (8.4\%) had sarcopenia. In the multivariate logistic regression analysis after

adjustment for age, BMI, and medications, by using non-frailty group as the reference, the

pre-frailty group was significantly more likely to have sarcopenia (OR: $2.77,95 \% \mathrm{Cl}: 1.05-9.26$,

$p=0.044$ ), but not cognitive decline or memory decline (Table 2 ). The frailty group was significantly

15 more likely to have cognitive decline (OR: 5.76, 95\% Cl: 1.20-27.6, $\mathrm{p}=0.029$ ), memory decline

16 (OR: 5.53, 95\% Cl: 1.64-18.7, p = 0.006), and sarcopenia (OR: 19.1, 95\% Cl: 3.73-98.0,

$17 \quad \mathrm{p}<0.001)($ Table 2).

18 In the logistic regression analysis in which the frailty groups were the dependent variables and

19 cognitive decline and sarcopenia were independent variables, cognitive decline was independently

20 only associated with a frailty score of $\geq 3$ (OR: $3.73,95 \% \mathrm{Cl}: 1.23-11.4, \mathrm{p}=0.020)$, while

21 sarcopenia was independently associated with both pre-frailty (score $\geq 1$; OR: 5.33, 95\% Cl: 
1 1.22-23.3, $p=0.026$ ) and frailty (score $\geq 3$; OR: $13.1,95 \% \mathrm{Cl}: 4.98-34.2, \mathrm{p}<0.001$ ). These

2 associations remained significant after adjustment for age and medications (Table 3).

3

4 


\section{Discussion}

2 The results of this study showed that frailty (defined as frailty score $\geq 3$ ) was associated with

3 cognitive decline, memory decline, and sarcopenia, and that pre-frailty (frailty score $=1$ or 2 ) was

4 associated with only sarcopenia. It is a new and interesting finding that there were differences in

5 the association between physical frailty and cognitive decline, memory decline, and sarcopenia

6 according to level of frailty.

$7 \quad$ In this study, we showed that frailty, but not pre-frailty, was associated with cognitive decline and

8 memory decline. Our results also showed that frailty and pre-frailty were associated with

9 sarcopenia, in contrast to cognitive and memory decline. In Japanese, multicenter,

10 population-based studies, the prevalence of dementia was not high among those aged

11 65-74 years (less than 10\%), but was higher among those aged 75 years and older ${ }^{24}$. The

12 prevalence of sarcopenia exhibited the same tendency, with the prevalence rising among those

13 aged 75 years and older 25,26 . Thus, older adults (particularly those 75 and older) are prone to both

14 cognitive impairment and sarcopenia. However, low physical performance, low physical strength,

15 and the decrease of muscle mass, which overlap with both sarcopenia and frailty, can be found

16 from middle age ${ }^{27-29}$. Thus, as shown in the results of this study, it is possible that sarcopenia is

17 associated with frailty at an earlier stage than is cognitive impairment, and that sarcopenia is

18 affected more by frailty than is cognitive impairment.

A recent study investigated the association of physical frailty and pre-frailty with dementia and 
1 Pre-frail older adults showed an increased risk for dementia in the aforementioned study, but some

2 estimates were not statistically significant in the fully adjusted models ${ }^{30}$. The results of that study

3 were consistent with our study. Previous studies indicated that frailty is associated with AD

4 pathology ${ }^{13}$ and biological mechanisms ${ }^{14}$, such as diffuse neuritic plaques, oxidative stress, and

5 inflammation. It is also possible that frailty and AD share common lifestyle risk factors, such as

6 physical inactivity and smoking, that lead to their pathophysiology, which contributes

7 simultaneously to physical frailty and $A D{ }^{13}$. On the other hand, it has been indicated that

8 comorbidities caused by cognitive impairment were also associated with frailty in $\mathrm{AD}$ or $\mathrm{MCl}$

9 patients ${ }^{31}$. Thus, it is likely that these associations interact with one another, leaving the causal

10 association between physical frailty and cognitive decline unclear. Further studies are required to

11 understand these associations.

12 Definitions of frailty and sarcopenia overlap, and sarcopenia is considered one of the core

13 symptoms of physical frailty ${ }^{5,6}$. The causal mechanisms underlying sarcopenia can be oxidative

14 stress, dysregulation of inflammatory cytokines and hormones, malnutrition, physical inactivity, and

15 muscle apoptosis, all of which have been hypothesized to contribute to frailty through interactive

16 pathways ${ }^{32,33}$. Recently, the definition of sarcopenia has been the coexistence of low muscle mass

17 and low physical performance 5, 20,34, which are contained in frailty domains. Thus, the association

18 of sarcopenia with even pre-frailty seems reasonable. Overlapping intervention strategies (e.g.,

19 nutritional supplementation and exercise) may be required to prevent both frailty and sarcopenia.

20 During recent years, the definition of frailty has been changing. Frailty has been considered to

21 include other aspects, for instance social aspects and comorbidities ${ }^{15}$. In addition to these aspects, 
1 poor cognition needs to be included in the definition of frailty, as shown in previous studies ${ }^{4,8}$ and

2 by this study. Furthermore, this study indicated that poor cognition was associated with frailty, and

3 that sarcopenia was associated even with pre-frailty. The results indicate that we need to

4 understand the consecutive mechanism as well as the association of pre-frailty and frailty with

5 cognitive decline, sarcopenia, and other adverse health outcomes. Interventions may need to be

6 tailored to the level of frailty to effectively prevent various functional declines. Future studies should

$7 \quad$ investigate these intervention strategies.

8 There were several limitations to this study. First, the cross-sectional design prevented us from

9 establishing causal associations between frailty and cognitive decline or sarcopenia. Second, the

10 findings in this study should be considered preliminary owing to the relatively small sample size,

11 which may introduce some error of inference, reduce the power of analysis, and limit generalization.

12 Third, the design of this study was not a population sampling, and participants in this study were

13 independent in ADLs. This may lead to an underestimation of the prevalence of frailty, cognitive

14 decline, and sarcopenia, as the participants were relatively healthy elderly persons.

15 In conclusion, our results indicate that there were differences in the association between physical

16 frailty and cognitive decline, memory decline, and sarcopenia according to the level of frailty.

17 Cognitive decline and memory decline were associated with frailty. Sarcopenia was associated with

18 pre-frailty and frailty. Further studies are required to understand these associations including

19 biological mechanisms. 


\section{Acknowledgements}

2 We would like to thank the students of the Department of Human Health Sciences at Kyoto

3 University for their help with data collection.

4

$5 \quad$ Conflict of Interest: The authors declare no conflicts of interest.

6 


\section{$1 \quad$ References}

2 1. Fried LP, Tangen CM, Walston J, et al. Frailty in older adults: evidence for a phenotype. J Gerontol A Biol

$4 \quad 2 . \quad$ Clegg A, Young J, Iliffe S, et al. Frailty in elderly people. Lancet. 2013;381:752-762.

5 3. Shimada H, Makizako H, Doi T, et al. Combined prevalence of frailty and mild cognitive impairment in a

4. Pel-Littel RE, Schuurmans MJ, Emmelot-Vonk MH, Verhaar HJ. Frailty: defining and measuring of a

5. Cruz-Jentoft AJ, Baeyens JP, Bauer JM, et al. Sarcopenia: European consensus on definition and diagnosis: report of the European Working Group on Sarcopenia in Older People. Age Ageing. 2010;39:412-423.

6. Xue $\mathrm{QL}$, Bandeen-Roche $\mathrm{K}$, Varadhan $\mathrm{R}$, et al. Initial manifestations of frailty criteria and the development of frailty phenotype in the Women's Health and Aging Study II. J Gerontol A Biol Sci Med Sci. 2008;63:984-990.

7. Ferrucci L, Guralnik JM, Studenski S, et al. Designing randomized, controlled trials aimed at preventing or delaying functional decline and disability in frail, older persons: a consensus report. J Am Geriatr Soc. 2004;52:625-634.

8. Searle SD, Mitnitski A, Gahbauer EA, et al. A standard procedure for creating a frailty index. $B M C$ Geriatr. 2008;8:24.

9. Fried LP, Ferrucci L, Darer J, et al. Untangling the concepts of disability, frailty, and comorbidity: implications for improved targeting and care. J Gerontol A Biol Sci Med Sci. 2004;59:255-263.

10. Mitnitski AB, Song X, Rockwood K. The estimation of relative fitness and frailty in community-dwelling older adults using self-report data. J Gerontol A Biol Sci Med Sci. 2004;59:M627-632.

11. Buchman AS, Boyle PA, Wilson RS, et al. Frailty is associated with incident Alzheimer's disease and cognitive decline in the elderly. Psychosom Med. 2007;69:483-489.

12. Boyle PA, Buchman AS, Wilson RS, et al. Physical frailty is associated with incident mild cognitive impairment in community-based older persons. J Am Geriatr Soc. 2010;58:248-255.

13. Buchman AS, Schneider JA, Leurgans S, Bennett DA. Physical frailty in older persons is associated with Alzheimer disease pathology. Neurology. 2008;71:499-504.

14. Mulero J, Zafrilla P, Martinez-Cacha A. Oxidative stress, frailty and cognitive decline. J Nutr Health Aging. 2011;15:756-760.

15. Morley JE, Vellas B, van Kan GA, et al. Frailty consensus: a call to action. J Am Med Dir Assoc. 2013;14:392-397.

16. Yesavage JA, Brink TL, Rose TL, et al. Development and validation of a geriatric depression screening scale: a preliminary report. $J$ Psychiatr Res. 1982;17:37-49.

17. Folstein MF, Folstein SE, McHugh PR. "Mini-mental state". A practical method for grading the cognitive state of patients for the clinician. $J$ Psychiatr Res. 1975;12:189-198.

18. Takechi H, Dodge HH. Scenery Picture Memory Test: a new type of quick and effective screening test to detect early stage Alzheimer's disease patients. Geriatr Gerontol Int. 2010;10:183-190.

19. Lezak MD, Howieson DB, Loring DW. Neuropsychological assessment. 4th ed. New York: Oxford Univ Pr. 2004. 
$120 . \quad$ Chen LK, Liu LK, Woo J, et al. Sarcopenia in Asia: consensus report of the asian working group for sarcopenia. J Am Med Dir Assoc. 2014;15:95-101.

21. Gibson AL, Holmes JC, Desautels RL, et al. Ability of new octapolar bioimpedance spectroscopy analyzers to predict 4-component-model percentage body fat in Hispanic, black, and white adults. Am J Clin Nutr. 2008;87:332-338.

22. Janssen I, Baumgartner RN, Ross R, et al. Skeletal muscle cutpoints associated with elevated physical disability risk in older men and women. Am J Epidemiol. 2004;159:413-421.

23. Janssen I. Influence of sarcopenia on the development of physical disability: the Cardiovascular Health Study. JAm Geriatr Soc. 2006;54:56-62.

24. Ikejima C, Hisanaga A, Meguro K, et al. Multicentre population-based dementia prevalence survey in Japan: a preliminary report. Psychogeriatrics. 2012;12:120-123.

25. Akune T, Muraki S, Oka H, et al. Exercise habits during middle age are associated with lower prevalence of sarcopenia: the ROAD study. Osteoporos Int. 2014;25:1081-1088.

26. Yamada M, Nishiguchi S, Fukutani N, et al. Prevalence of sarcopenia in community-dwelling Japanese older adults. JAm Med Dir Assoc. 2013;14:911-915.

27. Lauretani F, Russo CR, Bandinelli S, et al. Age-associated changes in skeletal muscles and their effect on mobility: an operational diagnosis of sarcopenia. J Appl Physiol (1985). 2003;95:1851-1860.

28. Speakman JR, Westerterp KR. Associations between energy demands, physical activity, and body composition in adult humans between 18 and 96 y of age. Am J Clin Nutr. 2010;92:826-834.

29. Yamada M, Moriguch Y, Mitani T, et al. Age-dependent changes in skeletal muscle mass and visceral fat area in Japanese adults from 40 to 79 years-of-age. Geriatr Gerontol Int. 2014;14 Suppl 1:8-14.

30. Kulmala J, Nykänen I, Mänty M, Hartikainen S. Association between Frailty and Dementia: a population-based study. Gerontology. 2014;60:16-21.

31. Ni Mhaolain AM, Gallagher D, Crosby L, et al. Correlates of frailty in Alzheimer's disease and mild cognitive impairment. Age Ageing. 2011;40:630-633.

32. Marcell TJ. Sarcopenia: causes, consequences, and preventions. J Gerontol A Biol Sci Med Sci. 2003;58:M911-916.

33. Dirks AJ, Hofer T, Marzetti E, et al. Mitochondrial DNA mutations, energy metabolism and apoptosis in aging muscle. Ageing Res Rev. 2006;5:179-195.

34. Morley JE, Abbatecola AM, Argiles JM, et al. Sarcopenia with limited mobility: an international consensus. J Am Med Dir Assoc. 2011;12:403-409.

3 
Table 1. Demographic differences according to frailty scores

\begin{tabular}{|c|c|c|c|c|c|c|}
\hline & \multirow[b]{2}{*}{$\begin{array}{c}\text { Total } \\
(n=273)\end{array}$} & \multicolumn{3}{|c|}{ Frailty level } & \multirow[b]{2}{*}{$\begin{array}{c}p \\
\text { for trend }\end{array}$} & \multirow[b]{2}{*}{ Post-hoc } \\
\hline & & $\begin{array}{l}\text { Non-frailty } \\
\qquad(\mathrm{n}=89)\end{array}$ & $\begin{array}{l}\text { Pre-frailty } \\
(n=155)\end{array}$ & $\begin{array}{l}\text { Frailty } \\
(n=29)\end{array}$ & & \\
\hline Age $(y)$ & $73.0 \pm 5.4$ & $73.1 \pm 4.6$ & $72.3 \pm 5.6$ & $76.6 \pm 5.1$ & $<0.001^{\dagger}$ & $a, b$ \\
\hline BMI $\left(\mathrm{kg} / \mathrm{m}^{2}\right)$ & $22.5 \pm 3.2$ & $22.2 \pm 3.0$ & $22.7 \pm 3.3$ & $21.9 \pm 3.8$ & 0.291 & - \\
\hline Medications & $2.32 \pm 2.24$ & $2.18 \pm 2.35$ & $2.23 \pm 2.10$ & $3.27 \pm 2.55$ & 0.072 & - \\
\hline Walking speed $(\mathrm{m} / \mathrm{s})$ & $1.40 \pm 0.20$ & $1.43 \pm 0.18$ & $1.41 \pm 0.20$ & $1.21 \pm 0.20$ & $<0.001^{\dagger}$ & $a, b$ \\
\hline Grip strength (kg) & $22.4 \pm 4.0$ & $23.4 \pm 3.4$ & $22.6 \pm 3.8$ & $18.3 \pm 4.1$ & $<0.001^{\dagger}$ & $a, b$ \\
\hline Cognitive decline (n) & $18(6.56 \%)$ & $4(4.49 \%)$ & $9(5.81 \%)$ & $5(17.2 \%)$ & $0.047^{*}$ & \\
\hline Memory decline (n) & $20(7.33 \%)$ & $6(6.74 \%)$ & $4(2.58 \%)$ & $10(34.5 \%)$ & $<0.001^{\dagger}$ & \\
\hline Sarcopenia (n) & $22(8.06 \%)$ & $2(2.25 \%)$ & 9 (5.81\%) & $11(37.9 \%)$ & $<0.001^{\dagger}$ & \\
\hline
\end{tabular}

$\mathrm{BMI}=$ body mass index

Non-frailty was defined as frailty score of 0 , pre-frailty was score 1 or 2 , frailty was score 3 or greater.

Cognitive decline was defined as the cut-off of MMSE score (23/24).

Memory decline was defined as the cut-off of SPMT score (9/10).

Sarcopenia was defined by using the AWGS-recommended diagnostic algorithm.

$*: p<0.05, \dagger: p<0.01$
a, significant difference between frailty and non-frailty $(p<0.01)$
b, significant difference between score frailty and pre-frailty $(p<0.01)$ 
Table 2. Relationship between the level of frailty and cognitive decline, memory decline, and sarcopenia

\begin{tabular}{|c|c|c|c|c|c|c|}
\hline \multirow[b]{2}{*}{ Frailty level } & \multicolumn{2}{|c|}{ Cognitive decline } & \multicolumn{2}{|c|}{ Memory decline } & \multicolumn{2}{|c|}{ Sarcopenia } \\
\hline & OR $(95 \% \mathrm{Cl})$ & $p$ & OR $(95 \% \mathrm{Cl})$ & $p$ & OR $(95 \% \mathrm{CI})$ & $p$ \\
\hline Non-frailty & 1 [Reference] & - & 1 [Reference] & - & 1 [Reference] & - \\
\hline Pre-frailty & $1.79(0.47-6.84)$ & 0.394 & $0.37(0.10-1.36)$ & 0.134 & $2.77(1.05-9.26)$ & $0.044^{*}$ \\
\hline Frailty & $5.76(1.20-27.6)$ & $0.029 *$ & $5.53(1.64-18.7)$ & $0.006^{\dagger}$ & $19.1(3.73-98.0)$ & $<0.001^{\dagger}$ \\
\hline
\end{tabular}

The analyses for cognitive decline and memory decline were adjusted for age, BMI, and medications.

The analysis for sarcopenia was adjusted for age and medications.

$*: p<0.05, \dagger: p<0.01$ 


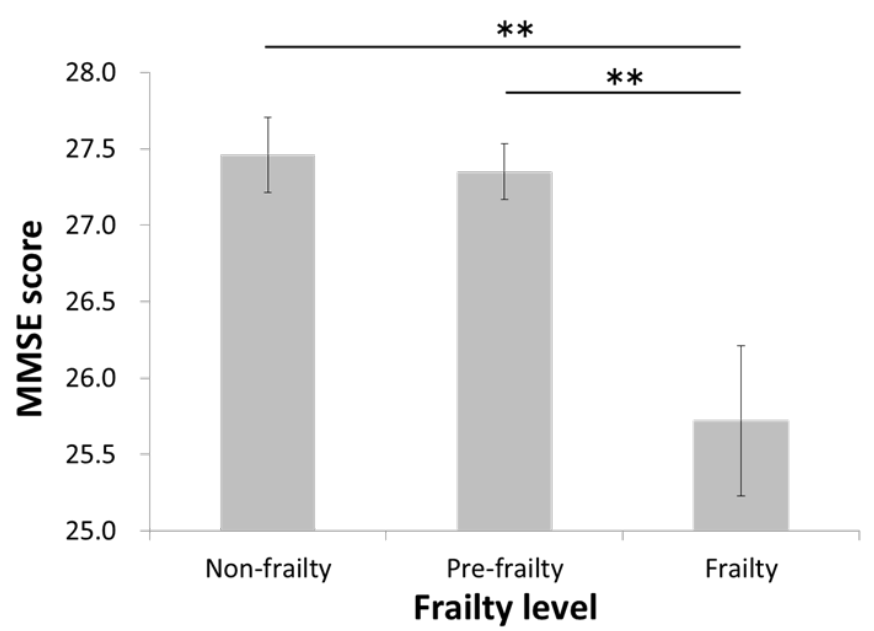

(a)

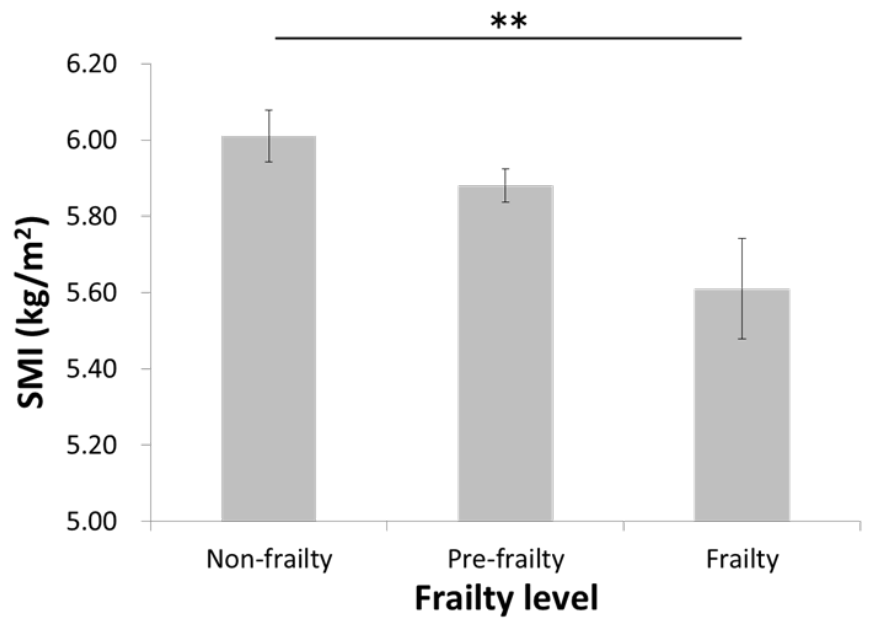

(c)

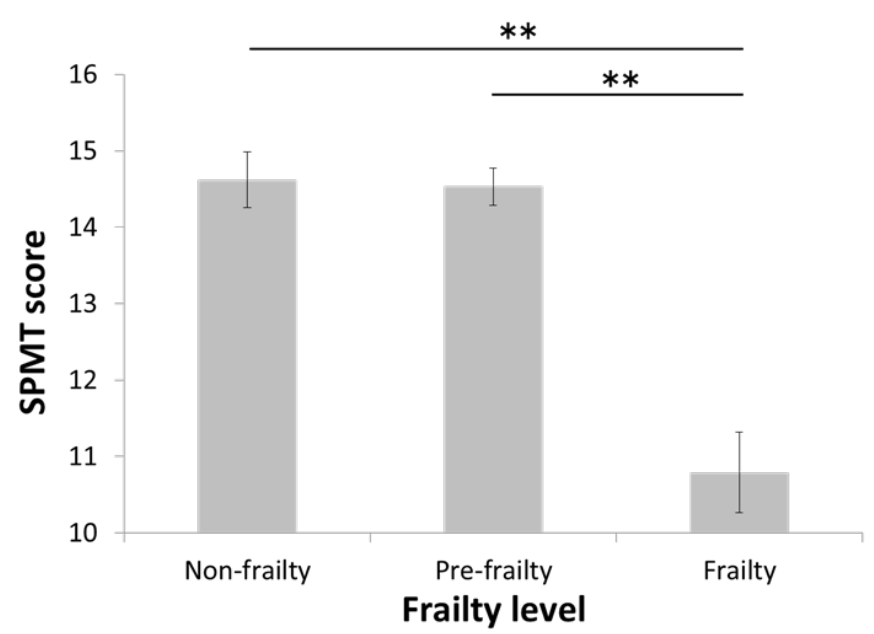

(b)

3 Figure 1. Comparison of the MMSE, SPMT, and SMI between the groups according to the level of

4 frailty.

5 (a) There were significant differences in the MMSE scores between the three groups $(F=6.78$,

$6 p=0.001)$.

7 (b) There were significant differences in the SPMT scores between the three groups $(F=18.5$,

$8 \quad p<0.001)$.

9 (c) There were significant differences in the SMI between the three groups $(F=5.17, p=0.006)$.

$\star: p<0.05, * *: p<0.01$ 